\title{
Comprensión docente de la relación entre aprendizaje matemático y funciones ejecutivas
}

\author{
José Hernando Ávila-Toscano 1*, Leonardo José Vargas-Delgado', Glenis Liliana Escobar-Pérez', \\ Adriana Paola Peñaloza-Torres ${ }^{\top}$ y Maura Alejandra Herrera-Bravo² \\ 1 Universidad del Atlántico \\ ${ }^{2}$ Corporación Universitaria Rreformada
}

Resumen: La Neurociencia cobra fuerza aplicada a las Matemáticas, destacando los estudios enfocados en funciones ejecutivas, que facilitan autorregulación, monitoreo, planeación y solución de problemas. No obstante, no se tiene certeza de que el profesorado comprenda la importancia de las funciones ejecutivas para la enseñanza de las Matemáticas. Este estudio enfocó su objetivo en determinar si, desde la comprensión de maestros y futuros maestros, las habilidades matemáticas y las funciones ejecutivas son variables independientes unas de otras, o si por el contrario pueden existir relaciones entre ellas. Con un diseño transversal, correlacional simple se evaluó 250 participantes mediante el Mathematics Skills Questionnaire (MSQ), analizando los datos mediante correlación canónica no lineal. Los participantes asignan importancia a la flexibilidad cognitiva y a las habilidades fácticas, conceptuales y procedimentales de las matemáticas. El análisis canónico destaca dos formas comprensivas de la relación entre las variables, comprensión integrativa y comprensión formalista. Se discuten las implicaciones de ambas en la enseñanzaaprendizaje de las Matemáticas.

Palabras clave: Enseñanza, Matemáticas, Funciones ejecutivas, Cualificación pedagógica, Habilidades matemáticas, Comprensión.

\section{Teachers' understanding of the relationship between mathematical learning and executive functions}

\begin{abstract}
Neuroscience is becoming more relevant applied to mathematics, highlighting studies focused on executive functions, which facilitate self-regulation, monitoring, planning and problem solving. However, it is not certain whether professors understand the importance of executive functions for the teaching of mathematics. This study focused on determining whether, in the understanding of teachers and future teachers, mathematical skills and executive functions are independent of each other, or whether, in contrast, they may be related to each other. With a simple cross-sectional, correlational design, 250 participants were evaluated using the Mathematics Skills Questionnaire (MSQ), analyzing the data through nonlinear canonical correlations. Participants assigned importance to cognitive flexibility and factual, conceptual, and procedural skills in mathematics. Canonical analysis highlights two comprehensive ways of relating variables, integrative understanding and formalistic understanding. The implications of both concepts for mathematical teaching-learning are discussed.
\end{abstract}

Keywords: Support, Significant Persons, Family, Friendships, Subjective Well-being.

Actualmente, la enseñanza de las Matemáticas privilegia competencias que permiten al estudiante reflexionar, crear, proponer soluciones a problemas y plantear la

Recibido: 06/10/2020 - Aceptado: 07/12/2020 - Avance online: 11/01/2021 *Correspondencia: José Hernando Ávila Toscano.

Universidad del Atlántico

Dirección: Barraquilla, Colombia.

E-mail: joseavila@mail.uniatlantico.edu.co

Ávila-Toscano, J. H., Vargas-Delgado, L. J., Escobar-Pérez, G. L., Peñaloza-Torres, A. P., y Herrera-Bravo, M. A. (2021). Comprensión docente de la relación entre aprendizaje matemático y funciones ejecutivas. Revista de Psicología y Educación, 16(1), 44-59, https:// doi.org/10.23923/rpye2021.01.201 comunicación de sus resultados, en oposición a tendencias previas basadas en estrategias memorísticas y aprendizaje algorítmico mecánico (Ministerio de Educación Nacional, 2006). Concordantes con este enfoque, en las últimas décadas la Educación se ha nutrido de diferentes aportes de campos de conocimientos como la Neurociencia, que vincula la funcionalidad cerebral con el 1699-9517/@ 2021 Asociación Científica de Psicología y Educación (ACIPE). Publicado por Consejo General de Colegios Oficiales de Psicólogos, España. Este es un artículo Open Access bajo la CC BY-NC-ND licencia (http://creativecommons.org/licencias/bync-nd/4.0/). 
aprendizaje, proveyendo un marco referencial soportado en resultados científicos de alto nivel que paulatinamente han contribuido a la reflexión pedagógica (Yoldi, 2015).

Para la Organización para la Cooperación y el Desarrollo Económico (OCDE, 2009), la Educación debe adaptarse al cerebro, pues no es posible esperar que los aprendices dominen información o procesos para los cuales no hay una adaptación cerebral. Dentro de los resultados de estudios neurocientíficos con mayor aplicación educativa destaca lo relativo a las funciones ejecutivas, siendo especialmente críticas para la lectoescritura y las Matemáticas (Roebers et al., 2012; Bernal y Rodríguez, 2014). Estas funciones son entendidas como habilidades que facilitan a los individuos el repertorio cognitivo requerido frente a la solución de un problema (Flores y Santos, 2017). También permiten la adaptabilidad, anulan pensamientos y respuestas automáticas y generan conductas dirigidas a metas (RojasBarahona, 2017).

Las funciones ejecutivas ayudan a monitorear y controlar el pensamiento y la acción. Las principales son inhibición (supresión de respuestas erróneas, resistir a actuar impulsivamente), memoria de trabajo (retención y evocación de información) y flexibilidad cognitiva (pensar creativamente, ver sucesos desde diferentes perspectivas y adaptarse rápido a circunstancias cambiantes) (Diamond, 2014; Gilmore y Cragg, 2014). Su desarrollo es gradual en concordancia con el proceso de maduración, entendido como todos los cambios que sufre el individuo gracias a la coordinación genética que predetermina ciclos de desarrollo biológico en momentos puntuales de la vida (Munakata et al., 2004).

La investigación destaca el rol de la educación formal para lograr que el estudiante adquiera y fortalezca los procesos de aprendizaje apelando al papel del funcionamiento ejecutivo, lo que permite al maestro el uso de estrategias cognitivas acordes con las exigencias educativas y al tiempo desarrollar en los estudiantes la capacidad de reflexionar y representar mentalmente los retos planteados (FloresLázaro, Castillo-Preciado et al., 2014). Cuando se aprovecha el funcionamiento ejecutivo en las diversas situaciones de enseñanza-aprendizaje, se genera un efecto positivo en múltiples escenarios que incluyen el plano interpersonal del estudiante, su rendimiento académico y una variedad de actividades de la vida cotidiana (Fuhs et al., 2014; Yoldi, 2015). Esto sugiere empezar su estimulación desde la escuela, destacando, por ejemplo, el desarrollo de la inhibición y la memoria de trabajo, ya que contribuyen con el rendimiento en pruebas matemáticas y su relación aumenta con el paso de los años (Cowan et al., 2011).

Las funciones ejecutivas y el aprendizaje matemático tienen una interacción permanente y el desarrollo de una tiene resultados positivos en la otra (Bull y Lee, 2014; Cantin et al., 2016). Hay evidencia que señala que las funciones ejecutivas son relevantes para adquirir conceptos y manejar operaciones matemáticas, como también para su mantenimiento y mejora (Risso et al., 2015). Es esperable el surgimiento de cambios con el paso del tiempo en la relación de estas variables, producto de los cambios cuantitativos y cualitativos del desarrollo, lo que implica la necesidad de un estudio constante de dicha relación (Cragg y Gilmore, 2014).

También se ha señalado que las funciones ejecutivas operan como facilitadoras en la decodificación de material numérico para su aprendizaje de memoria de forma sencilla. La flexibilidad facilita una comprensión profunda de hechos numéricos (Simanowski y Krajewski, 2017), pues permite seleccionar y aplicar a la resolución de problemas matemáticos la estrategia de solución más pertinente dentro del repertorio individual (Verschaffel et al., $2011)$. La inhibición está implicada en el uso o no de la intuición al resolver problemas matemáticos (Star y Pollack, 2015), o en tareas de precisión relacionadas con la comparación de fracciones (Gómez et al., 2015). Otras evidencias demuestran que así como se implican en el logro matemático, la afectación de las funciones ejecutivas incide 
en las dificultades en el área (Abreu-Mendoza et al., 2018; McDonal y Berg, 2017).

La literatura señala como conveniente vincular el desarrollo de las funciones ejecutivas en las actividades educativas, de forma que el conocimiento de los procesos cognitivos y su sustento cerebral puedan garantizar una pedagogía que aproveche las facultades personales y su contribución con el logro matemático (López et al., 2013). El papel del docente en este proceso es esencial (Cabanes et al., 2018), pues reconocer la interacción cerebro-aprendizaje puede ayudar a que las prácticas de enseñanza sean orientadas de forma propicia (Cragg et al., 2017).

La OCDE (2018) ha descrito al profesorado como el recurso de mayor relevancia en las escuelas, siendo además aquel en el que se concentra la mayor parte del gasto educativo materializado en salarios y procesos de formación. El organismo es enfático al afirmar que los mejores docentes son cruciales para el aprendizaje de los estudiantes en su aplicación del conocimiento a contextos reales y en los resultados para la vida. Contar con educadores interesados en el funcionamiento ejecutivo y capacitados para su fomento a través del acto pedagógico, constituye un aporte relevante para el desempeño educativo así como implicaciones de alto valor para la vida formativa, económica y social de los estudiantes (Fonseca et al., 2016; Pardos y González, 2018).

La comprensión docente de los procesos de aprendizaje resulta esencial a la hora de seleccionar herramientas y metodologías, establecer estrategias y fijar objetivos de aprendizaje, lo cual puede verse alimentado cuando el docente tiene conocimientos e interpreta los aspectos neurocientíficos que contribuyen al proceso de enseñanzaaprendizaje de las ciencias (Zárate, 2017). Sin embargo, a pesar del creciente desarrollo científico en la materia, en la práctica educativa todavía es poca la relevancia que se le brinda al papel de las funciones ejecutivas en el aprendizaje. El desconocimiento de los profesores en cuanto a esto es uno de los factores influyentes (Atmaca, 2016).
Es pertinente que la formación del maestro incluya conocimientos relativos al funcionamiento del cerebro y puntualmente de las funciones ejecutivas, deslindando la forma en la que inciden en el aprendizaje. Como señala Mason (2009), los fundamentos neurobiológicos de las funciones ejecutivas pueden ayudar a entender aún más el papel del efecto en el aprendizaje y abrir nuevas áreas de estudio. De hecho, las investigaciones en neurociencia recientemente han llamado la atención de los educadores, sin embargo, la forma como se aproximan docentes y futuros docentes hacia estos contenidos no siempre garantiza la completa aprehensión de su significado. Mason (2009) plantea que no deben fomentarse las aplicaciones directas de los hallazgos de Neurociencia a la educación, esto porque los neurocientíficos utilizan un lenguaje que apenas es entendido por los profesores y porque están demasiado centrados en el rigor de su investigación experimental, por lo tanto, no son los comunicadores más cualificados, además de que los docentes prefieren explicaciones más detalladas y contextualizadas.

Aunque son muchos los educadores interesados en la Neurociencia que creen que hay una conexión natural entre esta y la Educación, los profesores rara vez están expuestos a la evidencia de Neurociencia de origen primario - a los métodos neurocientíficos en su entrenamiento. En cambio, a menudo dependen de los resúmenes e interpretaciones de maestros especializados en el área del aprendizaje del cerebro (Coch y Ansari, 2009). Incluso, los educadores han informado que la complejidad y la orientación teórica de las investigaciones en Neuropsicología es problemática también señalan que el uso de lenguaje técnico y la estadística hacen que los hallazgos de la investigación sean difíciles de interpretar y, por lo tanto, no sean útiles para llevarlos al aula de clases (Edelenbosch et al., 2015). Estos hallazgos revelan que la utilización de la investigación neurocientífica en la Educación podría ser particularmente problemática, dadas las diferencias en terminología, metodología y estilos de presentación entre los dos campos (Laski et al., 2013). 
Tales aspectos limitan la aproximación del docente hacia contenidos neurocientíficos, de hecho, las fuentes existentes señalan que la mayoría de los maestros indican comprender de una $u$ otra forma el efecto que pueden tener procesos cerebrales en los logros académicos, sin embargo, su conocimiento sobre funciones ejecutivas sigue siendo intuitivo (Rapoportetal., 2016). En otros casos los docentes reconocen tener un papel importante en la promoción de habilidades ejecutivas, sin embargo, admiten tener dificultades en los conocimientos sobre funciones ejecutivas, especialmente por la compleja terminología que suele emplearse en los reportes neuropsicológicos (Keenan et al., 2020). También se ha reportado que la falta de comprensión del papel de estas funciones en el aprendizaje puede conducir a que los maestros realicen interpretaciones sesgadas del desempeño estudiantil. Borst y Houdé (2014) enfatizan el papel del control inhibitorio frente a este caso. Desde su punto de vista, enfocarse en la habilidad del niño o niña a partir del desempeño en una prueba es un indicador sesgado de su aprendizaje, dado que este no depende únicamente de los conocimientos y habilidades del estudiante sino también de su capacidad de inhibir reacciones inadecuadas que sirven de obstáculos para demostrar su conocimiento.

A pesar de los hallazgos sobre las implicaciones de las funciones ejecutivas en el aprendizaje, y particularmente en relación con las Matemáticas, en la actualidad es muy poca la evidencia sobre las formas de comprensión de los profesores acerca de la importancia de estas habilidades en el estudio matemático. Mucho menos esperable es la identificación de fuentes que aborden este fenómeno en docentes en proceso de formación. Algunos trabajos recalcan que los maestros en sus formas de comprensión otorgan mayor relevancia al papel de habilidades específicas en Matemáticas (saber hechos numéricos, conocer fórmulas, comprender conceptos) por encima de los aportes de las funciones ejecutivas para el aprendizaje de esta ciencia. Incluso, un número importante reconoce tener poca comprensión sobre el papel que desempeñan las habilidades ejecutivas, además de indicar que el conocimiento adquirido sobre el tema se debe a su propia experiencia docente y no a su formación profesional (Gilmore y Cragg, 2014).

También existe evidencia importante que demuestra cómo la comprensión del valor de las funciones ejecutivas en el aprendizaje es sustancial para la mejora educativa. De acuerdo con varios estudios, las estrategias de aula utilizadas por los docentes como forma de andamiaje de las funciones ejecutivas producen efectos valiosos en el desempeño de estas habilidades. Esto depende en gran medida de la calidad de las intervenciones y el apoyo emocional otorgado a los estudiantes, así como de los niveles de preparación y calidad escolar de los docentes en el aula (Choi et al., 2016; Hatfield et al., 2016). Opuestamente, cuando los docentes no desarrollan apropiadas formas de acompañamiento pedagógico, sus prácticas predicen de forma negativa el desempeño ejecutivo en niños y niñas (Bardack y Obradović, 2019).

De acuerdo con lo expuesto, este estudio se enfoca en determinar si desde la comprensión de maestros y futuros maestros las habilidades matemáticas y las funciones ejecutivas son dos conjuntos de variables independientes uno del otro o si por el contrario pueden existir relaciones entre los dos.

\section{MÉTODO}

\section{PARTICIPANTES}

La muestra estuvo conformada por 250 personas (127 hombres, 123 mujeres), con media de edad de 27.9 (DT =9.0). De estas 250 personas, 129 eran docentes de Matemáticas en ejercicio (72 hombres, 57 mujeres), con edad promedio de 31.6 años $(D T=10.1)$; y 121 eran estudiantes de Licenciatura en Matemáticas (55 hombres, 66 mujeres), es decir, formándose para ser docentes del área, quienes tuvieron media de edad de 24.1 años (DT $=5.5$ ).

Todos los participantes se seleccionaron en la ciudad de Barranquilla (Colombia) mediante muestreo no probabilístico de forma incidental, considerando como criterio 
de inclusión para el caso de los maestros, que estuvieran debidamente titulados y en ejercicio de su tarea docente en el área de las Matemáticas a nivel escolar. Para los docentes en formación, considerando que su programa de estudios dura cuatro años divididos en ocho semestres académicos, se fijó como criterio de inclusión que cursaran de cuarto semestre en adelante, esto con el fin de garantizar que tuvieran mayor apropiación de los procesos relacionados con la enseñanza y el aprendizaje de la matemática de manera que la falta de conocimiento previo no sesgara los resultados. escolar. El cuestionario consta de tres dominios específicos relacionados con el aprendizaje de las Matemáticas: Funciones Ejecutivas ( $\alpha=.73$ ), enfocado en evaluar memoria de trabajo, flexibilidad cognitiva e inhibición; Habilidades Matemáticas $(\alpha=.56)$, que se refieren al conocimiento conceptual, procedimental y fáctico, así como la distinción entre habilidades abstractas y concretas; y Otras Habilidades ( $\alpha$ $=.81$ ), las cuales, aunque no son específicas de las Matemáticas, se relacionan con el logro en esta área e incluyen habilidades verbales, espaciales y la creatividad (Gilmore y Cragg, 2014).

Tabla 1

Dominios y elementos del Mathematics Skills Questionnaire (MSQ)

\begin{tabular}{|c|c|}
\hline Dominio & Habilidades \\
\hline \multirow{4}{*}{ Funciones Ejecutivas } & Manipular información abstracta \\
\hline & Almacenar y manipular información mentalmente \\
\hline & Centrarse en la información relevante y evitar distracciones \\
\hline & Ser capaz de pensar con flexibilidad \\
\hline \multirow{4}{*}{ Habilidades Matemáticas } & Saber hechos numéricos \\
\hline & Comprender conceptos matemáticos \\
\hline & Comprender cómo se usan las matemáticas en el mundo real \\
\hline & Conocer fórmulas y procedimientos \\
\hline \multirow{4}{*}{ Otras Habilidades } & Tener buenas habilidades verbales \\
\hline & Ser capaz de proporcionar razones para respaldar sus soluciones \\
\hline & Ser capaz de pensar creativamente \\
\hline & Tener buenas habilidades espaciales \\
\hline
\end{tabular}

\section{INSTRUMENTOS}

Se empleó una ficha demográfica para recoger información relacionada con edad, sexo y tipo de participante (docente en ejercicio o en formación). Además se aplicó el instrumento denominado Mathematics Skills Questionnaire (MSQ) (Tabla 1), creado por Gilmore y Cragg (2014), para medir la importancia de habilidades básicas para el aprendizaje de Matemáticas según maestros del área de cualquier grado
El cuestionario pide al evaluado calificar la importancia de las 12 habilidades en una escala tipo Likert de 5 puntos $(0=$ No importante, 4 =Extremadamente importante). Los diferentes tipos de elementos están mezclados en el cuestionario y para su valoración los docentes deben responder a la pregunta "Para ser buenos en matemáticas en la escuela ¿̇ué tan importante cree que es para los estudiantes cada una de las siguientes habilidades?" 


\section{PROCEDIMIENTO}

Los instrumentos se organizaron en un formulario web y se realizó la evaluación por vía electrónica. El formulario era anónimo, no se hizo registro de correos electrónicos para garantizar la confidencialidad además de respetar las disposiciones legales colombianas en torno al manejo de información personal. El cuestionario estuvo abierto por espacio de un mes para su diligenciamiento, además se garantizó que el enlace de acceso al formulario estuviera restringido a la admisión de una sola respuesta por persona.

\section{ANÁLISIS DE DATOS}

Se desarrolló un estudio de corte transversal, con estrategia asociativa y diseño relacional simple (Ato et al., 2013), puesto que busca identificar relaciones funcionales entre variables sin que exista manipulación de estas. El estudio implicó también la comparación de grupos naturales con el objetivo de encontrar diferencias significativas entre estos (Shaughnessy et al., 2012). Los grupos fueron representados por docentes en ejercicio y docentes en formación.

Tras recoger la información, se comprobaron las propiedades psicométricas del instrumento mediante análisis factorial exploratorio, dado que no contaba con aplicación previa en población colombiana, por ello se optó por este análisis en lugar del confirmatorio. Revisadas las propiedades de la prueba, se procedió a identificar la importancia relativa otorgada por los participantes a cada conjunto de habilidades estudiadas, para ello se analizaron los resultados de forma descriptiva y se comparó cada conjunto de habilidades entre los participantes (docentes en ejercicio y en formación) empleando la U de Mann-Whitney. Este estadístico se utilizó en virtud de que las variables no cumplieron el supuesto de normalidad. El tamaño del efecto se probó con la $r$ de Rosenthal ( $r=.10$ pequeño; $r$ $=.30$ medio; $r=.50$ grande).

Para probar la independencia de habilidades matemáticas y funciones ejecutivas, se aplicó correlación canónica no lineal, dado que opera como un tipo de extensión de la regresión múltiple pero en este caso permite identificar en qué medida dos conjuntos de variables categóricas muestran similitud. Su ventaja radica en que el segundo conjunto contiene más de una variable de respuesta que pueden tener niveles de medición nominal, ordinal o numérico (Meulman y Heiser, 2005). Todas las variables se escalaron con nivel de medición ordinal, en virtud de la escala gradada del instrumento aplicado. El tipo de participante se empleó como variable de etiquetado para la generación de gráficos de puntuaciones de objeto. Todos los procedimientos se cumplieron con el software SPSS v.23.0 (IBM Corp., 2015).

\section{RESULTADOS}

En primer lugar se analizaron las propiedades del MSQ, con el fin de garantizar que la medición de las variables se ajustara a los dominios definidos por Gilmore y Cragg (2014). Se siguió el mismo procedimiento de las autoras mediante exploración de ítems por dominio con análisis de componentes principales, para determinar si los reactivos eran retenidos en una sola dimensión.

Todos los ítems cargaron en un solo dominio para el caso de las Funciones Ejecutivas $(\mathrm{KMO}=.621$; Bartlett $=120.024, \mathrm{p}=.001)$ $y$ de Otras Habilidades (KMO =.788; Bartlet $t=291.422, p=.001)$; mientras que en Habilidades Matemáticas se requirió la eliminación de un ítem ("Comprender cómo se usan las Matemáticas en el mundo real"), dado que cargaba para un segundo componente de único ítem. La solución final fue un dominio de tres reactivos (KMO $=.630$; Bartlett $=66.591, p=.001$ ). Las puntuaciones de importancia media para los 11 ítems finales del instrumento, junto con sus respectivas cargas, se describen en la Tabla 2.

La varianza de cada uno de los dominios mostró algunas variaciones en relación con la prueba original, en ese caso cada factor único representaba el 44\%, 55\% y $65 \%$ 
Tabla 2

Comparación descriptiva de puntuaciones medias (máximo 4) y carga factorial de los ítems del MSQ en el estudio actual frente al de Gilmore y Cragg (2014).

\begin{tabular}{|c|c|c|c|c|c|}
\hline \multicolumn{2}{|c|}{$\begin{array}{l}\text { Dimensiones evaluadas e ítems que les } \\
\text { componen }\end{array}$} & \multicolumn{2}{|c|}{ Gilmore y Cragg (2014) } & \multicolumn{2}{|c|}{ Elaboración propia } \\
\hline Dominio & Ítems & Media & $\begin{array}{l}\text { Carga } \\
\text { factorial }\end{array}$ & Media & $\begin{array}{l}\text { Carga } \\
\text { factorial }\end{array}$ \\
\hline \multirow{3}{*}{$\begin{array}{l}\text { Habilidades } \\
\text { matemáticas } \\
\text { específicas }\end{array}$} & Saber hechos numéricos & 3.29 & .76 & 3.11 & .75 \\
\hline & $\begin{array}{l}\text { Comprender conceptos } \\
\text { matemáticos }\end{array}$ & 3.38 & .61 & 3.56 & .74 \\
\hline & $\begin{array}{l}\text { Conocer fórmulas y } \\
\text { procedimientos }\end{array}$ & 3.00 & .70 & 2.95 & .70 \\
\hline \multirow{4}{*}{ Funciones Ejecutivas } & $\begin{array}{l}\text { Manipular información } \\
\text { abstracta }\end{array}$ & 2.64 & .76 & 3.04 & .73 \\
\hline & $\begin{array}{l}\text { Almacenar y manipular } \\
\text { información mentalmente }\end{array}$ & 2.68 & .74 & 2.89 & .53 \\
\hline & $\begin{array}{l}\text { Centrarse en la información } \\
\text { relevante y evitar distracciones }\end{array}$ & 3.05 & .67 & 3.24 & .72 \\
\hline & $\begin{array}{l}\text { Ser capaz de pensar con } \\
\text { flexibilidad }\end{array}$ & 3.02 & .80 & 3.44 & .71 \\
\hline \multirow{4}{*}{ Otras habilidades } & $\begin{array}{l}\text { Tener buenas habilidades } \\
\text { verbales }\end{array}$ & 2.53 & .86 & 3.12 & .79 \\
\hline & $\begin{array}{l}\text { Ser capaz de proporcionar } \\
\text { razones para respaldar sus } \\
\text { soluciones }\end{array}$ & 3.22 & .82 & 3.47 & .80 \\
\hline & $\begin{array}{l}\text { Ser capaz de pensar } \\
\text { creativamente }\end{array}$ & 2.76 & .78 & 3.54 & .81 \\
\hline & $\begin{array}{c}\text { Tener buenas habilidades } \\
\text { espaciales }\end{array}$ & 2.48 & .75 & 3.24 & .74 \\
\hline
\end{tabular}

para los dominios Habilidades Matemáticas, Funciones Ejecutivas y Otras Habilidades respectivamente; mientras que en el presente estudio los porcentajes de la varianza total explicada fueron de $54.2 \%, 46.6 \%$ y $62.3 \%$ para los mismos dominios. Los valores de consistencia interna de las puntuaciones del instrumento en este estudio correspondieron a $\alpha=.56, \alpha=.60$ y $\alpha=.78$, muy parecidos al trabajo original, lo que según Gilmore y Cragg (2014) puede deberse a la naturaleza multicomponente de la habilidad matemática.

Para dar cumplimiento al objetivo trazado, inicialmente se procedió a identificar el nivel de importancia concedido por docentes y futuros docentes de Matemáticas a cada conjunto de habilidades frente al aprendizaje del área. En la Tabla 3 se presentan los datos de resumen de las variables. En ellas se aprecia el porcentaje de respuesta para cada categoría, con valores elevados en las categorías Extremadamente importante y Muy importante para Habilidades Matemáticas como "comprensión de conceptos matemáticos", "saber hechos numéricos" y "conocer fórmulas y procedimientos". Entre tanto, en la importancia atribuida a las Funciones Ejecutivas también se hallaron valores altos, aunque también son las habilidades donde se reporta mayor cantidad de respuestas No importante y Algo importante. De todas las Funciones Ejecutivas, la de mejor valoración es la flexibilidad, mientras que en el dominio Otras Habilidades sobresalió la creatividad ("ser capaz de pensar creativamente"), la capacidad argumentativa ("ser capaz de proporcionar razones para respaldar sus soluciones") y las habilidades espaciales ("tener buenas habilidades espaciales"). 
Tabla 3

Estadísticos descriptivos correspondientes al nivel de importancia de cada habilidad evaluada por docentes y futuros docentes $(N=250)$.

\begin{tabular}{|c|c|c|c|c|c|c|c|}
\hline & \multicolumn{2}{|c|}{$M(D T)$} & \multicolumn{5}{|c|}{ Nivel de importancia (\%) } \\
\hline & Docente & $\begin{array}{l}\text { Futuro } \\
\text { docente }\end{array}$ & $\mathrm{NI}$ & Al & 1 & $\mathrm{Ml}$ & El \\
\hline \multicolumn{8}{|l|}{ Habilidades matemáticas } \\
\hline Saber hechos numéricos & $3.08(0.87)$ & $3.14(0.77)$ & 0.8 & 3.6 & 13.6 & 48.0 & 34.0 \\
\hline $\begin{array}{l}\text { Comprender conceptos } \\
\text { matemáticos }\end{array}$ & $3.57(0.64)$ & $3.55(0.60)$ & 0.0 & 0.8 & 4.8 & 31.6 & 62.8 \\
\hline $\begin{array}{l}\text { Conocer fórmulas y } \\
\text { procedimientos }\end{array}$ & $2.98(0.83)$ & $2.91(0.86)$ & 0.0 & 4.8 & 24.4 & 42.0 & 28.8 \\
\hline \multicolumn{8}{|l|}{ Funciones ejecutivas } \\
\hline Manipular información abstracta & $3.10(0.77)$ & $2.97(0.80)$ & 0.4 & 2.4 & 2.0 & 47.6 & 29.6 \\
\hline $\begin{array}{l}\text { Almacenar y manipular } \\
\text { información mentalmente }\end{array}$ & $2.84(0.95)$ & $2.95(0.89)$ & 0.8 & 5.2 & 28.0 & 36.0 & 3.0 \\
\hline $\begin{array}{l}\text { Centrarse en la información } \\
\text { relevante y evitar distracciones }\end{array}$ & $3.36(0.70)$ & $3.11(0.84)$ & 0.4 & 1.2 & 15.6 & 4.0 & 42.8 \\
\hline $\begin{array}{l}\text { Ser capaz de pensar con } \\
\text { flexibilidad }\end{array}$ & $3.47(0.65)$ & $3.42(0.69)$ & 0.0 & 0.8 & 7.6 & 38.0 & 53.6 \\
\hline \multicolumn{8}{|l|}{ Otras habilidades } \\
\hline $\begin{array}{l}\text { Tener buenas habilidades } \\
\text { verbales }\end{array}$ & $3.19(0.85)$ & $3.05(0.98)$ & 2.0 & 3.2 & 15.2 & 39.6 & 4.0 \\
\hline $\begin{array}{l}\text { Ser capaz de proporcionar } \\
\text { razones para respaldar sus } \\
\text { soluciones }\end{array}$ & $3.54(0.63)$ & $3.39(0.71)$ & 0.0 & 1.6 & 5.6 & 37.2 & 55.6 \\
\hline $\begin{array}{l}\text { Ser capaz de pensar } \\
\text { creativamente }\end{array}$ & $3.58(0.60)$ & $3.50(0.69)$ & 0.0 & 1.2 & 5.2 & 31.6 & 62.0 \\
\hline $\begin{array}{l}\text { Tener buenas habilidades } \\
\text { espaciales }\end{array}$ & $3.36(0.72)$ & $3.12(0.78)$ & 0.4 & 1.6 & 12.8 & 43.6 & 41.6 \\
\hline
\end{tabular}

La comparación de las medias entre los dos grupos de participantes (docentes y futuros docentes) no mostró diferencias significativas en relación las Habilidades Matemáticas ("comprensión de conceptos matemáticos" $U$ $=7532.5, p=.57$; "saber hechos numéricos" $U=76.02, p=.70$; "conocer fórmulas y procedimientos" $U=7459.0, p=.52$ ), lo que sugiere porcentajes similares en la evaluación de la importancia relativa de dichas habilidades. Por su parte, se hallaron diferencias con efecto pequeño y mayor suma de rangos entre los docentes titulares frente a las Funciones Ejecutivas "inhibición" $(U=6579.0, p=.02, r$ $=.15)$ y "habilidades espaciales" ( $U=6387.0$, $p=.01, r=.17)$, que forman parte del conjunto de variables Otras Habilidades.
Para determinar si, desde la comprensión de los dos grupos de participantes, Habilidades Matemáticas y Funciones Ejecutivas son dos conjuntos de variables independientes o no, se procedió a aplicar un análisis de correlación canónica. Se probaron varios modelos con soluciones tri y bidimensionales, incluyendo también las variables correspondientes al dominio Otras Habilidades. Tras evaluar los modelos, se optó por una solución bidimensional empleando Habilidades Matemáticas y Funciones Ejecutivas, al ser la de mejor ajuste. Los resultados fueron etiquetados de acuerdo con el tipo de participante. 
El modelo final mostró un estadístico de ajuste con valor de 1.724 y una media de pérdidas de 0.276, lo que demuestra un muy buen comportamiento. El autovalor de la primera dimensión equivale a 0.998 , lo que explica $58 \%$ (autovalor/ajuste total) del juste real del modelo. Por su parte, en la segunda dimensión el autovalor es de 0.726 , explicando $42 \%$ del ajuste total del modelo. En la Tabla 4 se recogen todos los estadísticos de ajuste obtenidos en la correlación canónica donde se observa que la solución bidimensional ofrece resultados óptimos, ubicando cuatro variables en la primera dimensión y tres en la segunda. Adicionalmente, los estadísticos de pérdida simple refuerzan la apropiada selección y escalamiento de las variables al identificarse valores cercanos a cero.
Posteriormente se otorgó una denominación a cada dimensión surgida de la correlación canónica, con el fin de definir los tipos de comprensión surgidos acorde con las relaciones identificadas entre ambos conjuntos de variables. La primera dimensión fue denominada comprensión integrativa, dado que integra variables de ambos conjuntos, y corresponde a participantes que presentan un nivel de comprensión por el cual se asume que el desempeño ejecutivo está implicado en el aprendizaje de las Matemáticas por su relación con habilidades matemáticas puntuales. En este caso destaca como principal habilidad disciplinar la comprensión de conceptos matemáticos, lo cual se relaciona con funciones ejecutivas como la flexibilidad cognitiva, el control inhibitorio y con la manipulación de información abstracta.

Tabla 4

Estadísticos de ajuste en el análisis de correlación canónica entre los conjuntos de variables Habilidades Matemáticas y Funciones Ejecutivas ( $N=250)$.

\begin{tabular}{|c|c|c|c|c|c|c|c|c|c|}
\hline \multirow{3}{*}{ Variables analizadas a } & \multicolumn{3}{|c|}{ Ajuste múltiple } & \multicolumn{3}{|c|}{ Ajuste simple } & \multicolumn{3}{|c|}{ Pérdida simple } \\
\hline & \multicolumn{2}{|c|}{ Dimensión } & \multirow[b]{2}{*}{ Suma } & \multicolumn{2}{|c|}{ Dimensión } & \multirow[b]{2}{*}{ Suma } & \multicolumn{2}{|c|}{ Dimensión } & \multirow[b]{2}{*}{ Suma } \\
\hline & 1 & 2 & & 1 & 2 & & 1 & 2 & \\
\hline Saber hechos numéricos & .001 & .168 & .168 & .001 & .166 & .166 & .000 & .002 & .002 \\
\hline Comprender conceptos matemáticos & .988 & .011 & 1.000 & .988 & .011 & .999 & .000 & .000 & .000 \\
\hline Conocer fórmulas y procedimientos & .001 & .415 & .416 & .001 & .415 & .416 & .000 & .000 & .000 \\
\hline 2 Manipular información abstracta & .504 & .064 & .568 & .502 & .015 & .517 & .001 & .050 & .051 \\
\hline Almacenar y manipular información & .000 & .621 & .621 & .000 & .618 & .618 & .000 & .003 & .003 \\
\hline Centrarse en la información relevante & .515 & .004 & .519 & .515 & .000 & .515 & .000 & .004 & .004 \\
\hline Ser capaz de pensar con flexibilidad & .990 & .054 & 1.044 & .990 & .041 & 1.031 & .000 & .013 & .013 \\
\hline $\begin{array}{l}\text { a Nivel de escalamiento óptimo ordinal. } \\
\text { Fuente: elaboración propia. }\end{array}$ & & & & & & & & & \\
\hline
\end{tabular}


La segunda dimensión fue denominada comprensión formalista enfocada en el objeto matemático, por cuanto privilegia dominios específicos de este tipo de conocimiento. Esta dimensión resalta habilidades matemáticas que se centran en el conocimiento de fórmulas y procedimientos, así como en hechos numéricos, las cuales interactúan con la capacidad de almacenamiento y manipulación de información, es decir, el uso de procesos memorísticos. Salvo el papel mnémico en esta dimensión no se valoran las demás funciones ejecutivas.

Finalmente, en la Figura 1 se presentan las puntuaciones de objeto etiquetadas de acuerdo con el tipo de participante. Esta información deja observar que no se define un grupo puntual de acuerdo con las dimensiones de comprensión obtenidas, sino que en ambas dimensiones es posible identificar la participación de docentes titulares y docentes en formación.

\section{DISCUSIÓN}

Este estudio se realizó con el objetivo de determinarsi, desde la comprensión de maestros y futuros maestros, habilidades matemáticas y funciones ejecutivas son dos conjuntos de variables independientes 0 relacionadas. Los resultados responden en primer lugar, al reconocimiento de la valoración otorgada a las habilidades analizadas, resaltando que tanto estudiantes como docentes les dan valoraciones similares a las habilidades matemáticas evaluadas, generalmente estimadas como muy importantes o extremadamente importantes por lo que para ambos grupos son relevantes. Sobresale entre todas ellas la comprensión de conceptos matemáticos como la habilidad de mayor importancia, así mismo destaca la ausencia de diferencias en la valoración otorgada por docentes en ejercicio frente a los que adelantan su formación docente, lo cual sugiere que ambos grupos evalúan de forma similar las habilidades analizadas.

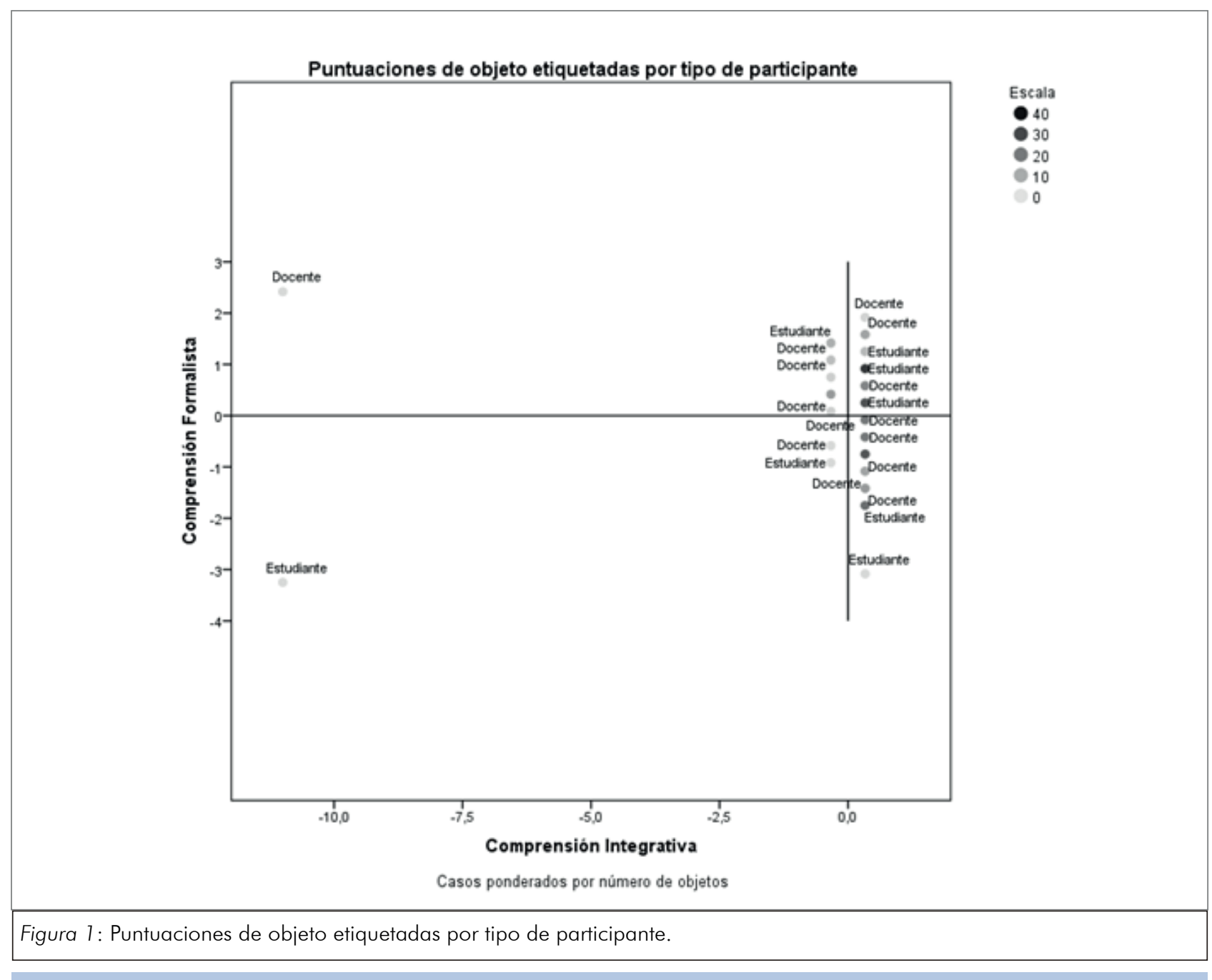


Una situación similar se registró al analizar la relevancia que asignan ambos grupos de participantes al papel de las funciones ejecutivas en el aprendizaje de las Matemáticas, puesto que nuevamente se reportaron altos valores en las categorías Muy importante y Extremadamente importante, sobresaliendo en este caso la flexibilidad mental como la de mejor valoración. En estas habilidades sí se reportó diferencias entre docentes y futuros docentes, puesto que en el primer grupo se observó una mayor importancia otorgada a la capacidad de centrarse en la información relevante y evitar distracciones.

Análogamente, al valorar las habilidades transversalestambién se identificaron resultados favorables para todas ellas. Incluso se registró diferencias en relación con las habilidades espaciales que fueron de mayor relevancia para el grupo de profesores en ejercicio. En cierta medida, estos datos contrastan con los previamente reportados por Gilmore y Cragg (2014), quienes señalaron que los maestros suelen identificar como elementos relevantes las habilidades específicas en Matemáticas por encima de las que corresponde a las funciones ejecutivas. En el caso de este estudio, al menos descriptivamente se observa que ambos grupos incluyen los tres conjuntos de habilidades como relevantes, si bien es cierto que los registros que asignan poca importancia a las variables estudiadas son mayores al valorar las habilidades que no son estrictamente matemáticas (véase Tabla 3).

Por otra parte, en cuanto a la consideración de aspectos relativos a la neurociencia y la educación, es interesante destacar que el nivel de conocimiento e interés sobre asuntos neurocientíficos suele no ser elevado entre el profesorado dedicado a la enseñanza de las matemáticas. Trabajos antecedentes (Gilmore y Cragg, 2014; Rapoport et al., 2016) han enfatizado en este tópico al indicar que es común que el conocimiento de los maestros sobre las FE se deba a su propia experiencia docente en lugar de derivarse de su formación universitaria, por lo que el conocimiento de los profesores sobre FE obedece en gran medida a la intuición y a su experiencia (Mason, 2009).
Hay que mencionar además que el poco interés en Neurociencias aplicadas a la Educación puede estar relacionado con el hecho de que no es común que el profesorado manipule de forma directa evidencia neurocientífica o conozca los métodos de investigación empleados en estas disciplinas. Esto hace que las formas de comunicación basadas en tecnicismos, estadísticas y sustratos anatómicos generen dificultades en la interpretación para los maestros (Cochy Ansari, 2009; Laski et al., 2013; Mason, 2009). Esto puede incidir en la abstención tanto de búsqueda de información relevante como de la participación e interpretación de investigaciones sobre las aplicaciones de la Neurociencia en el aula. Tal falta de interés implicaría además que los docentes no desarrollen competencias que les permitan incorporar y aplicar estos fundamentos para facilitar tanto la enseñanza como el aprendizaje. Con ello, podría verse afectado también el desarrollo del profesorado en términos de innovación, creatividad, y criticidad en el acto docente (Bardack y Obradović, 2019), aspectos que brindan los avances de la Neurociencia en la Educación.

Por otro lado, el hallazgo fundamental de este estudio surgió al determinar si desde la perspectiva de maestros y futuros maestros existía relación entre las habilidades matemáticas y las funciones ejecutivas, lo que facilitó inferir la forma de comprensión de los participantes sobre el papel de estas últimas en el aprendizaje de la Matemática. Los resultados refuerzan la idea de que efectivamente ambos conjuntos de variables están relacionados y podrían definir dos formas diferentes de comprender las implicaciones de unas con otras.

Inicialmente destaca la forma de comprensión que hemos denominado como integrativa, en la cual parece asumirse que las funciones ejecutivas están implicadas de forma relevante en el aprendizaje de las Matemáticas, relacionándose específicamente la comprensión de conceptos matemáticos con la flexibilidad cognitiva, el control inhibitorio y la manipulación abstracta. A partir de los resultados pareciera ser que los participantes 
con este tipo de comprensión reconocen que las funciones ejecutivas benefician la enseñanza-aprendizaje en educación matemática. Al incluir el control inhibitorio podría pensarse que desde esta perspectiva se asume que esta función ejecutiva permite a los estudiantes inhibir acciones, pensamientos y emociones que no convienen o no aportan a la comprensión de conceptos matemáticos, mientras que la flexibilidad cognitiva ayudaría a cambiar o adaptar los conocimientos, posibilitando modificar o redirigir los planes del aprendizaje.

La segunda forma de comprensión la hemos definido como de tipo formalista, y de ella puede inferirse que prioriza el interés por el objeto matemático (Cowan et al., 2011 ) dada la prelación de habilidades formales como el conocimiento de fórmulas, procedimientos y hechos numéricos, que además se relacionan con la capacidad de almacenamiento y manipulación de información.

En esta dimensión se toma únicamente las habilidades de la memoria de trabajo sobre las otras habilidades de las funciones ejecutivas como complemento que interactúa con las habilidades matemáticas, lo que sugiere que esto le permitiría al estudiante mantener en mente la información relevante para las Matemáticas e ir actualizándola, evaluarla y manipularla en beneficio de su aprendizaje en esta área. Claramente se trata de una visión mucho más tradicionalista tanto de la Matemática como de su proceso de aprendizaje.

Esta segunda perspectiva puede sugerir que entre el profesorado (incluido aquel en formación) existen concepciones tradicionales sobre la enseñanza y el aprendizaje de la ciencia matemática. Por lo cual constituye un reto en el marco de las políticas nacionales de Educación (MEN, 2006, 2016), trabajar desde el saber pedagógico y didáctico en la transformación de las concepciones relativas a los contenidos científicos, entendiendo las implicaciones de múltiples habilidades que superan lo fáctico y lo procedimental, así como en la inclusión de posturas interdisciplinarias que enriquezcan la forma de comprender la matemática y redunden en procesos de calidad para su enseñanza en actividades propias del aula.

Coincidimos con estudios antecedentes (Coch y Ansari, 2009; Dubinsky et al, 2013; Choi et al., 2016; Hatfield et al., 2016), en recomendar que la formación de futuros maestros debería contemplar la orientación en cursos sobre Neurociencia Cognitiva, - bien propender por la integración de su formación actual con los hallazgos surgidos del estudio del cerebro en relación con el aprendizaje (Ansari, Cochy De Smedt, 2011). Esto implica la apropiación concienzuda de tales fundamentos para evitar interpretaciones erróneas (Varas-Genestiera y Ferreira, 2017), siguiendo planes de desarrollo en el aula que garanticen una interacción funcional entre docentes y estudiantes. La investigación en la materia viene reforzando la importancia de generar interacciones que ayudan a potencializar el desempeño ejecutivo que impacta de forma directa en el aprendizaje de ciencias como la Matemática (Bardack y Obradović, 2019).

El presente estudio afronta algunas limitaciones, por un lado se trató de una muestra de tipo incidental a la que se accedió por medio de herramientas electrónicas, lo que reduce la participación a la disponibilidad e interés personal al momento de cumplir el cuestionario. Un tamaño de muestra más amplio también es apetecible para futuros estudios. Adicionalmente, los valores obtenidos en los coeficientes de consistencia de las puntuaciones fueron bajos para las habilidades matemáticas. Si bien esto es un dato reconocido por las autoras del instrumento y atribuido al carácter multicomponente del saber matemático (Gilmore y Cragg, 2014), exige prudencia en la interpretación de algunos resultados.

La comprensión de la Matemática como conocimiento científico demanda de apertura cognitiva para incluir múltiples perspectivas que inciden en su proceso de enseñanza y aprendizaje. Las funciones ejecutivas constituyen habilidades con suficientes pruebas empíricas que demuestran su aporte al desarrollo de las capacidades de los estudiantes en la disciplina matemática, lo que 
revela el valor agregado de aplicar estrategias de aula que estimulen el desarrollo ejecutivo. Esto no implica que el educador se transforme en un experto en Neurociencias, ni despersonalizar la Educación como la conocemos, sino propender por formas holísticas de entendimiento sobre los elementos que median el aprendizaje matemático redundando en nuevas didácticas que reconozcan el papel de procesos inhibitorios y de flexibilidad cognitiva puesto que ofrecen buenos resultados en el desempeño matemático.

Las formas de comprensión que tiene el docente sobre la participación de las funciones ejecutivas en el aprendizaje de la Matemática son susceptibles de influir sobre su actuar metodológico. Este será el resultado de concebir apropiadamente los problemas y considerar las acciones que debe realizar el estudiante en el aula para la planeación, ejecución y control de las actividades. Es decir, dichas funciones son relevantes en el marco de la Educación, ya que están vinculadas al buen desempeño académico en áreas de alto progreso en el aula, como la Matemática. Acorde con esta postura, se evidencia que el conocimiento de los maestros en ejercicio y en formación sobre el debido funcionamiento ejecutivo de los estudiantes, puede tener un impacto positivo en el aprendizaje y el desempeño académico, lo que refleja la importancia y conectividad con el campo de la Neurociencia, sus derivados y la educación matemática.

- Conflicto de intereses.

Los autores declaran no tener ningún conflicto de intereses.

\section{REFERENCIAS}

Abreu-Mendoza, R.A., Chamorro, Y., GarcíaBarrera, M.A., \& Matute, E. (2018). The contributions of executive functions to mathematical learning difficulties and mathematical talent during adolescence. PLoS ONE 13(12), e0209267. https://doi. org/10.1371/iournal.pone.0209267

Ato, M., López, J., \& Benavente, A. (2013). Un sistema de clasificación de los diseños de investigación en Psicología. Anales de Psicología, 29(3), 1038-1059. https://doi. org/10.6018/analesps.29.3.178511
Atmaca, Ç. (2016). Brain-based learning practices of teacher educators in an English language teaching programme. Electronic Turkish Studies, 17(9), 101-134. https:// doi.org/10.7827/TurkishStudies.9084

Ansari, D., Coch, D., \& De Smedt, B. (2011). Connecting education and cognitive neuroscience: Where will the journey take us? Educational Philosophy and Theory, 43(1), 37-42. https://doi. org/10.1111/i.1469-5812.2010.00705.

Bernal, F., \& Rodríguez, M. (2014). Estimulación temprana de las funciones ejecutivas en escolares, una revisión actualizada. Orientación Educacional, 28(53), 15-24.

Bardack, S., \& Obradović, J. (2019). Observing teachers' displays and scaffolding of executive functioning in the classroom context. Journal of Applied Developmental Psychology, 62, 205-219. https://doi. org/10.1016/i.appdev.2018.12.004

Borst, G., \& Houdé, O. (2014). Inhibitory control as a core mechanism for cognitive development and learning at school. Perspectives on Languaje and Lliteracy, 40(2), 41-44.

Bull R., \& Lee, K. (2014). Executive functioning and mathematics achievement. Child Development Perspectives, 8(1), 36-41. https://doi.org/10.1111/cdep.12059

Cantin, R., Gnaedinger, E., Gallaway, K., Hessen-Mclnnis, M., \& Hund., A. (2016). Executive functioning predicts reading, mathematics, and theory of mind during the elementary years. Journal of Experimental Child Psychology, 146, 66-78. https://doi. org/10.1016/i.jecp.2016.01.014

Choi, J. Y., Castle, S., Williamson, A. C., Young, E., Worley, L., Long, M., \& Horm, D. M. (2016). Teacher-child interactions and the development of executive function in preschool-age children attending head start. Early Education and Development, 27(6), 751-769. https://doi.org/10.1080 /10409289.2016.1129864

Cragg, L., Keeble, S., Richardson, S., Roome, H.E., \& Gilmore, C. (2017). Direct and indirect influences of executive functions on mathematics achievement. Cognition, 162, 12-26. https://doi.org/10.1016/i. cognition.2017.01.014 
Cragg, L., \& Gilmore, C. (2014). Skills underlying mathematics: The role of executive function in the development of mathematics proficiency. Trends in Neuroscience and Education, 3(2), 63-68. https://doi.org/10.1016/i. tine.2013.12.001 i

Cabanes, L., Colunga, S., \& García, J. (2018). Funciones ejecutivas en escolares: un programa de intervención desde la matemática. Opuntia Brava, 9(2), 59-73. https://doi.org/10.35195/ob.v9i2. 148

Cowan, R., Donlan, C., Shepherd, D.L., Cole-Fletcher, R., Saxton, M., \& Hurry, J. (2011). Basic calculation proficiency and mathematics achievement in elementary school children. Journal of Educational Psychology, 103, 786-803. https://doi. $\mathrm{org} / 10.1037 / \mathrm{a} 0024556$

Coch, D., \& Ansari, D. (2009). Thinking about mechanisms is crucial to connecting neuroscience and education. Cortex, 45(4), 546-547. https://doi.org/10.1016/i. cortex.2008.06.001

Diamond, A. (2014). Executive functions: Insights into ways to help more children thrive. Zero to three, 35(2), 9-17.

Dubinsky, J.M., Roehrig, G., \& Varma, S. (2013). Infusing neuroscience into teacher professional development. Educational Researcher, 42(6), 317-329. https://doi. org/10.3102/0013189X13499403

Edelenbosch, R., Kupper, F., Krabbendam, L., \& Broerse, J.E.W. (2015). Brain-based learning and educational neuroscience: boundary work. Mind, Brain, and Education, 9(1), 40-49. https://doi.org/10.1111/ mbe. 12066

Fonseca, G.P., Rodríguez, L.C., \& Parra, J.H. (2016). Relación entre funciones ejecutivas y rendimiento académico por asignaturas en escolares de 6 a 12 años. Hacia la Promoción de la Salud, 21 (2), 41-58. https://doi.org/10.17151/ hpsal.2016.21.2.4

Flores, L.C., \& Santos, S.C. (2017). La Matemática en el desarrollo cognitivo y metacognitivo del escolar primario. Edusol, 17(60), 45-59. Disponible en https://bit. ly/2YFlg 42

Flores-Lázaro, J.C., Castillo-Preciado, R.E., \& Jiménez-Miramonte, N.A. (2014).
Desarrollo de funciones ejecutivas, de la niñez a la juventud. Anales de Psicología, 30(2), 463. https://doi.org/10.6018/ analesps.30.2.155471

Fuhs, M.W., Nesbitt, K.T., Farran, D.C., \& Dong, N. (2014). Longitudinal associations between executive functioning and academic skills across content areas. Developmental Psychology, 50(6), 1698-1708. https://doi.org/10.1037/ a0036633

Gilmore, C., \& Cragg, C. (2014). Teachers' Understanding of the role of executive functions in mathematics learning. Mind, Brain, and Education, 8(3), 132-136. https://doi.org/10.1111/mbe.12050

Gómez, D.M., Jiménez, A., Bobadilla, R., Reyes, C., \& Dartnell, P. (2015). The effect of inhibitory control on general mathematics achievement and fraction comparison in middle school children. ZDM, 47(5), 801811 . https://doi.org/10.1007/s 11858 015-0685-4

Hatfield, B.E., Burchinal, M.R., Pianta, R.C., \& Sideris, J. (2016). Thresholds in the association between quality of teacher-child interactions and preschool children's school readiness skills. Early Childhood Research Quarterly, 36(3), 561-571. https://doi. org/10.1016/i.ecresq.2015.09.005

IBM Corp. (2015). IBM SPSS Statistics for Windows, Version 23.0. Armonk, NY: IBM Corp.

Keenan, L., O'Sullivan, A., \& Downes, M. (2020). Teachers' experiences and understanding of executive functions in Irish primary school classrooms: Findings from a mixed-methods questionnaire. Irish Educational Studies, 1-14. https://doi.org/ 10.1080/03323315.2020.1794927

López, L.S., Ávila, M., \& Camargo, G. (2013). Atención selectiva y funciones ejecutivas como predictores del conocimiento matemático informal. Actas del VII CIBEM, 2723-2730. Montevideo. Disponible en https://bit.ly/2BOFdsT

Laski, E.V., Reeves, T.D., Ganley, C.M., \& Mitchell, R. (2013). Mathematics teacher educators' perceptions anduse of cognitive research. Mind, Brain, and Education, 7, 63-74. https://doi.org/10.1111/ mbe. 12009 
Mason., L. (2009). Bridging neuroscience and education: A two-way path is possible. Cortex, 45(5), 548-549. https://doi. org/10.1016/i.cortex.2008.06.003.

McDonald, P., \& Berg, D. (2017). Identifying the nature of impairments in executive functioning and working memory of children with severe difficulties in arithmetic. Child Neuropsychology, 28(4), 1047-1062. https://doi.org/10.1080/09297049.201 $\underline{7.1377694}$

Meulman, J., \& Heiser, W. (2005). SPSS Categories ${ }^{\circledR}$ 14.0. Chicago, USA: SPSS Inc.

Ministerio de Educación Nacional (MEN) (2006). Documento de fundamentación de los Derechos Básicos de Aprendizaje (V2) y de las Mallas de Aprendizaje Componente Matemáticas. Bogotá: MEN.

Ministerio de Educación Nacional (MEN) (2016). Estándares para la excelencia de la educación. Bogotá: MEN.

Munakata Y, Casey, B.J., \& Diamond, A. (2004). Developmental cognitive neuroscience: progress and potential. Trends in Cognitive Science, 8(3), 122-128. https://doi.org/10.1016/i. tics.2004.01.005

Organización para la Cooperación y el Desarrollo Económico (OCDE) (2009). La comprensión del cerebro. El nacimiento de una ciencia del aprendizaje. Santiago: Ediciones Universidad Católica Silva Henríquez.

Organización para la Cooperación y el Desarrollo Económico (OCDE) (2018). Effective teacher policies: Insights from PISA. PISA, OCDE Publishing. https://doi. org/10.1787/9789264301603-en

Pardos, A., \& González, M. (2018). Intervención sobre las Funciones Ejecutivas desde el contexto educativo. Revista Iberoamericana de Educación, 78(1), 27-42. https://doi.org/10.35362/ rie7813269

Risso, A., García, M., Durán, M., Brenlla, J.C., Peralbo, M., \& Barca, A. (2015). Un análisis de las relaciones entre funciones ejecutivas, lenguaje y habilidades matemáticas. Revista de Estudios e Investigación en Psicología y Educación, 9, 73-78
Roebers, C.M., Cimeli, P., Röthlisberger, M., \& Nevenschwander, R. (2012). Executive functioning, metacognition, and selfperceived competence in elementary school children: An explorative study on their interrelations and their role for school achievement. Metacognition and Learning, 7(3), 151-173. https://doi.org/10.1007/ s1 1409-012-9089-9

Rapoport, S., Rubinsten, O., \& Katzir, T. (2016). Teachers' beliefs and practices regarding the role of executive functions in reading and arithmetic. Frontiers in Psychology, 7, 1567. https://doi. org/10.3389/fpsyg.2016.01567

Rojas-Barahona, C.A. (2017). Funciones ejecutivas y educación: Comprendiendo habilidades clave para el aprendizaje. Santiago, Chile: Ediciones Universidad Católica de Chile.

Shaughnessy, J., Zechmeister, E., \& Zechmeister, J. (2012). Research methods in psychology (9th Ed.). New York, NY: McGraw-Hill.

Simanowski, S. \& Krajewski, K. (2017). Specific preschool executive functions predict unique aspects of mathematics development: A 3-year longitudinal study. Child Development, 90(3), 544-561. https://doi.org/10.1111/cdev.12909

Star, J.R., \& Pollack, C. (2015). Inhibitorycontrol and mathematics learning: definitional and operational considerations. ZDM, 47(5), 859-863. https://doi.org/10.1007/ s1 1858-015-0716-1

Varas-Genestiera, P., \& Ferreira, R.A. (2017). Neuromitos de los profesores chilenos: orígenes y predictores. Estudios Pedagógicos, 43(3), 341 360. https://doi.org/10.4067/s071807052017000300020

Verschaffel, L., Luwel, K., Torbeyns, J., \& Van Dooren, W. (2011). Analyzing and developing strategy flexibility in mathematics education. En J. Elen, E. Stahl, R. Bromme \& G. Clarebout (Eds.) Links between beliefs and cognitive flexibility. (pp. 175-197). Springer, Dordrecht. https://doi.org/10.1007/978-94-0071793-0 10

Yoldi, A. (2015). Las funciones ejecutivas: hacia prácticas educativas que potencien 
su desarrollo. Páginas de Educación, 8(1), $1-13,72-98$.

Zárate, M.B.C. (2017). El curriculum y las prácticas pedagógicas del docente de educación superior desde los aportes de la neurociencia. Revista Internacional de apoyo a la inclusión, logopedia, sociedad y multiculturalidad, 3(4), 1-11. 